\title{
DETERMINAÇÃO DE Fe, Zn, Cu E Mn EXTRAÍDOS DO SOLO POR DIFERENTES EXTRATORES E DOSADOS POR ESPECTROFOTOMETRIA DE EMISSÃO ÓTICA EM PLASMA INDUZIDO E ESPECTROFOTOMETRIA DE ABSORÇÃO ATÔMICA ${ }^{(1)}$
}

\author{
João José de Miranda Milagres ${ }^{(2)}$, Víctor Hugo Alvarez V ${ }^{(3,4)}$, Reinaldo \\ Bertola Cantarutti $^{(3)} \&$ Júlio César Lima Neves ${ }^{(3)}$
}

\begin{abstract}
RESUMO
Para aumentar a precisão nas análises químicas de fertilidade do solo e dosar simultaneamente vários elementos, alguns laboratórios vêm optando pelo uso da técnica da espectrofotometria de emissão ótica em plasma induzido (ICP), em detrimento da técnica da espectrofotometria de absorção atômica (EAA), hoje comumente utilizada nos laboratórios de análise de solos. Este trabalho, além de comparar as duas técnicas de dosagem quanto à precisão, à reprodutibilidade e à magnitude dos teores dos micronutrientes $\mathrm{Fe}, \mathrm{Zn}, \mathrm{Cu}$ e $\mathrm{Mn}$, extraídos por Mehlich-1, Mehlich-3 e DTPA-TEA, objetivou, também, selecionar os comprimentos de onda que apresentam menores interferências espectrais no ICP. Foram utilizadas 36 amostras (0 a 0,2 m) de solos coletadas nos Estados de Minas Gerais e Bahia, com ampla variação nos teores de micronutrientes, sendo selecionados três solos para definir os comprimentos de onda do ICP e avaliar a precisão e a reprodutibilidade dos métodos de dosagem. Os comprimentos de onda com menores interferências espectrais no ICP foram: $259,939 \mathrm{~nm}$ para Fe em Mehlich-1 e DTPA-TEA e 234,349 nm em Mehlich-3; 213,857 nm para $\mathrm{Zn}$ e 324,752 $\mathrm{nm}$ para $\mathrm{Cu}$ nos três extratores; e 259,372 $\mathrm{nm}$ para Mn em Mehlich-1 e DTPA-TEA e 260,568 $\mathrm{nm}$ em Mehlich-3. Tanto o ICP quanto o EAA foram precisos e reprodutíveis nas dosagens de Fe e Mn, sendo o ICP, em virtude do seu menor limite de detecção, mais preciso e reprodutível nas dosagens de $\mathrm{Zn}$ e $\mathrm{Cu}$. Os métodos de dosagem diferiram estatisticamente $(p<0,01)$ pelo teste de identidade aplicado, para as dosagens de
\end{abstract}

\footnotetext{
(1) Parte da Tese de Mestrado do primeiro autor apresentada à Universidade Federal de Viçosa - UFV. Trabalho desenvolvido com suporte financeiro da FAPEMIG. Recebido para publicação em janeiro de 2005 e aprovado em janeiro de 2007.

(2) Engenheiro-Agrônomo do Departamento de Solos, Universidade Federal de Viçosa - UFV. CEP 36570-000 Viçosa (MG). E-mail: jmilagres@ufv.br

(3) Professor do Departamento de Solos, UFV. E-mails: vhav@ufv.br; cantarutti@ufv.br; julio_n2003@yahoo.com.br

(4) Bolsista do CNPq.
} 
Fe, Zn, Cu e Mn, utilizando Mehlich-1, Mehlich-3 e DTPA-TEA, comprometendo assim a interpretação dos resultados gerados pelo ICP, com base nos níveis críticos gerados a partir do EAA.

Termos de indexação: análise química do solo, ICP, comparação de métodos, precisão, reprodutibilidade.

\title{
SUMMARY: DETERMINATION OF SOIL-EXTRACTED FE, ZN, CU AND MN BY DIFFERENT EXTRACTORS BY INDUCTIVELY COUPLED PLASMA OPTICAL EMISSION SPECTROMETRY AND ATOMIC ABSORPTION SPECTROMETRY
}

\begin{abstract}
In an attempt to enhance the precision of chemical analyses of soil fertility and quantify different elements simultaneously, some laboratories have opted to use the technique of inductively coupled plasma for optical emission spectrometry (ICP) instead of the technique of Atomic Absorption Spectrometry (AAS), which currently is the most commonly used in soil analysis laboratories. In this study we compared the precision of the two dosage techniques, their repeatability and the magnitude of the concentrations of the micronutrients $\mathrm{Fe}, \mathrm{Zn}, \mathrm{Cu}$ and $\mathrm{Mn}$, extracted through Mehlich-1, Mehlich-3 and DTPA-TEA; Additionaly, we aimed to select the wavelengths of lowest spectral interferences with the ICP. Thirtysix soil samples (0 to $0.2 \mathrm{~m}$ ) from the states of Minas Gerais and Bahia, Brazil, with varied micronutrient concentrations were used. To define the ICP wavelengths and to evaluate the precision and repeatability of the dosage methods, three of the 36 collected soils were selected. The wavelengths with the lowest spectral interferences in the ICP were: $259.939 \mathrm{~nm}$ for Fe in Mehlich-1 and DTPA-TEA and $234.349 \mathrm{~nm}$ in Mehlich-3; $213.857 \mathrm{~nm}$ for $\mathrm{Zn}$ and $324.752 \mathrm{~nm}$ for $\mathrm{Cu}$ in the three extractors; and 259.372 $\mathrm{nm}$ for Mn in Mehlich-1 and DTPA-TEA and $260.568 \mathrm{~nm}$ in Mehlich-3. Both ICP and AAS were precise and reproducible for $\mathrm{Fe}$ and $\mathrm{Mn}$ dosage, while ICP, owing to the lower detection limit, was more precise and reproducible in the $\mathrm{Zn}$ and $\mathrm{Cu}$ dosages. The dosage methods differed statistically $(p<0.01)$ by the identity test applied for the $\mathrm{Zn}, \mathrm{Cu}$, and $\mathrm{Mn}$ dosages, using Mehlich-1, Mehlich-3 and DTPA-TEA, which affected the interpretation of the ICP results, based on the critical levels of $A A S$.
\end{abstract}

Index terms: chemical soil analysis, ICP, comparison of methods, precision, reproducibility.

\section{INTRODUÇÃO}

No Brasil, as determinações dos micronutrientes $\mathrm{Fe}, \mathrm{Zn}, \mathrm{Cu}$ e Mn nas análises de rotina em fertilidade do solo apresentam, basicamente, dois procedimentos de extração. Em um desses procedimentos, utiliza-se o extrator Mehlich-1 (Defilippo \& Ribeiro, 1997), também utilizado para $\mathrm{P}$ e K disponíveis (CFSRS/SC, 1994; CFSEMG, 1999). O outro procedimento, usado no Estado de São Paulo, extrai Fe, Zn, Cu e Mn com DTPA-TEA (Raij et al., 1996). Um terceiro procedimento de extração vem sendo utilizado por instituições de pesquisa e consiste no uso do extrator Mehlich-3 (Mehlich, 1984).

O método de dosagem de micronutrientes utilizado pela maioria dos laboratórios é o mesmo: espectrofotometria de absorção atômica (EAA).

Na perspectiva de aumentar a precisão, baixar os limites de detecção e dosar simultaneamente vários elementos, algumas instituições de pesquisa e alguns laboratórios particulares estão optando pela espectrofotometria de emissão ótica em plasma induzido (Inductively Coupled Plasma-Optical Emission Spectrometry-ICP-OES ou, simplesmente, ICP) para dosagem de elementos em extratos de solos e plantas.

A utilização do método da espectrofotometria de emissão ótica em plasma induzido nas análises de rotina intensificou-se a partir da década de 1970, devido, principalmente, aos avanços tecnológicos direcionados para as fontes de excitação e para as aplicações computacionais. Segundo Raij et al. (2001), o plasma é a fonte de excitação mais efetiva para fins analíticos, sendo o plasma de argônio o mais utilizado. Plasmas, por definição, são gases em que uma significativa fração de átomos e moléculas apresentase ionizada (Soltanpour et al., 1982).

O princípio geral do método baseia-se na energização do átomo, que resulta no movimento de elétrons de um orbital mais próximo para outro mais 
afastado do núcleo, deixando o átomo num estado excitado. Quando a energização é elevada, transformando o átomo num íon, diz-se que ele atingiu o estado de ionização. Após o processo de excitação, os elétrons dos átomos excitados e, ou, dos íons excitados retornam rapidamente ao orbital de origem, emitindo energia eletromagnética, fótons, com comprimento de onda específico para cada elemento e cada transição. No espectrofotômetro, os fótons são transformados em sinais eletrônicos, que são convertidos em concentração, após as devidas calibrações (Boss \& Fredeen, 1997). No ICP é permitido trabalhar com um conjunto de comprimentos de onda selecionado para cada elemento. Dependendo do comprimento de onda escolhido, pode haver, ou não, interferências espectrais de outros elementos químicos, provocando distorções nos resultados analíticos. A interferência é detectada quando o pico de emissão (espectro) do elemento analisado não se apresenta uniforme.

Soltanpour et al. (1979), comparando os métodos ICP e EAA para dosagem de Fe, Zn, Cu e Mn extraídos com $\mathrm{NH}_{4} \mathrm{HCO}_{3}$-DTPA, verificaram que os valores para a declividade de uma reta $\left(\beta_{1}\right)$ não foram significativamente diferentes de 1 , exceto para $\mathrm{Cu}$ (teor médio de $1,8 \mathrm{mg} \mathrm{dm}^{-3}$ ), e que os valores do intercepto $\left(\beta_{0}\right)$ foram muito próximos de zero para Zn e Mn, muito pequenos para Fe e relativamente grandes para $\mathrm{Cu}$.

Resultados semelhantes entre os métodos ICP e EAA, para as dosagens de $\mathrm{Cu}, \mathrm{Zn}, \mathrm{Fe}, \mathrm{Mn}, \mathrm{Pb}$ e Cd, foram encontrados em 11 sedimentos e um solo da Austrália, após digestão em ácido nítrico concentrado (Willett \& Zarcinas, 1986).

Os teores dos micronutrientes Fe, Zn, Cu e Mn de 471 amostras de solos da região oeste dos EUA, extraídos por DTPA-TEA e dosados pelo ICP, foram comparados aos teores extraídos por Mehlich-3 e dosados por EAA, constatando-se que os métodos de dosagem não diferiram significativamente, indicando que o ICP pode ser utilizado como alternativa ao EAA (Vocasek \& Friedericks, 1994).

O uso da técnica ICP para gerar resultados a serem interpretados, com base nos níveis críticos gerados a partir da técnica EAA, torna-se preocupante. Constata-se, com isso, a necessidade de comparação entre os métodos ICP e EAA, utilizando os métodos de extração de micronutrientes, em análise química de fertilidade dos solos, adotados no Brasil.

Neste trabalho, objetivou-se comparar as técnicas de dosagem ICP e EAA quanto à precisão, à reprodutibilidade e à magnitude dos teores dos micronutrientes $\mathrm{Fe}, \mathrm{Zn}, \mathrm{Cu}$ e $\mathrm{Mn}$, extraídos por Mehlich-1, Mehlich-3 e DTPA-TEA, bem como selecionar no ICP os comprimentos de onda que apresentam menores interferências espectrais.

\section{MATERIAL E MÉTODOS}

O trabalho foi realizado nos laboratórios do Departamento de Solos da Universidade Federal de Viçosa. Foram selecionadas 36 amostras de solos da camada superficial (0-0,2 m), coletadas nos Estados de Minas Gerais e Bahia, sendo 30 de Latossolos, uma de Cambissolo, uma de Chernossolo, uma de Vertissolo, uma de Planossolo, uma de Neossolo e uma de Argissolo. Todas as análises deste estudo foram realizadas nos laboratórios do Departamento de Solos da Universidade Federal de Viçosa. A caracterização, por Mehlich-1, revelou ampla variação nos teores de $\mathrm{Fe}, \mathrm{Zn}, \mathrm{Cu}$ e Mn (Quadro 1).

$\mathrm{Na}$ fase de extração dos micronutrientes foram empregados os extratores Mehlich-1 (Defilipo \& Ribeiro, 1997), Mehlich-3 (Mehlich, 1984) e DTPA-TEA (Raij et al., 1996). Na fase de dosagem, utilizaram-se um espectrofotômetro de absorção atômica, marca GBC, modelo 908 AA, e um espectrofotômetro de emissão ótica em plasma induzido, marca Perkin Elmer, modelo Optima 3300 DV.

Para definição dos comprimentos de onda no ICP, foram testadas, inicialmente, todas as possibilidades disponibilizadas pelo aparelho, tanto no plano de visão axial quanto no plano radial. Procedeu-se então, para cada elemento, ao traçado do espectro de uma solução

Quadro 1. Valores (mínimo, médio e máximo), desvio-padrão e coeficiente de variação dos teores de Fe, Zn, $\mathrm{Cu}$ e Mn por Mehlich-1 dos 36 solos analisados

$\begin{array}{llll}\mathrm{Fe} & \mathrm{Zn} & \mathrm{Cu} & \mathrm{Mn}\end{array}$

$\begin{array}{lcccc}\text { Mínimo } & 66 & 0,0 & 0,0 & 0 \\ \text { Médio } & 354 & 4,6 & 1,6 & 60 \\ \text { Máximo } & 1.520 & 32,0 & 13,5 & 366 \\ \text { Desvio-padrão } & 269,3 & 7,36 & 2,58 & 77,4 \\ \text { CV (\%) } & 76 & 162 & 161 & 130\end{array}$


contendo apenas a espécie química a ser determinada, a fim de identificar e ajustar o pico de emissão do elemento. Posteriormente, fez-se a leitura do branco, de uma amostra e do ponto máximo da curva de calibração, a fim de verificar possíveis interferências espectrais e, se possível, minimizá-las, fazendo-se ajustes nas linhas de base, delimitando a área do pico. Após esses ajustes, fez-se a leitura da curva de calibração, selecionando-se os comprimentos de onda que apresentaram o coeficiente de determinação do modelo linear $\left(\mathrm{R}^{2}\right)$ mais próximo de 1 e com o mínimo de interferência espectral.

Utilizando três amostras de três solos diferentes (A, B e C) que apresentaram ampla variação nos teores dos micronutrientes e três repetições de cada amostra por série, selecionou-se, após 10 séries, para cada elemento, um único comprimento de onda, escolhido pelo critério da ausência ou da mínima interferência espectral, com boa precisão e reprodutibilidade. A opção por apenas um comprimento de onda por elemento e apenas um plano de visão objetivou reduzir o tempo de leitura e de interpretação de espectros, minimizando o consumo de gás e, conseqüentemente, diminuindo o custo da análise.

Para cada extrator, foram feitos novos ajustes, seguindo os mesmos procedimentos descritos anteriormente.

Para avaliação da precisão, que diz respeito à repetibilidade dentro de uma série de análises, e da reprodutibilidade, que se refere à medida da aproximação dos resultados médios obtidos com o mesmo método e a mesma amostra sob diferentes séries de análises, foram realizadas análises dos três solos (A, B e C), ou seja, os mesmos solos utilizados na definição dos comprimentos de onda. Os tratamentos corresponderam aos três solos, que foram analisados com três repetições por série, num total de 10 séries estratificadas, no tempo de $24 \mathrm{~h}$. Nos extratos dos solos foram realizadas as dosagens de $\mathrm{Fe}, \mathrm{Zn}, \mathrm{Cu}$ e Mn. Para cada elemento foi calculado o coeficiente de variação, que representou a precisão, quando se utilizou o desvio-padrão calculado dentro da série, e a reprodutibilidade, quando se utilizou o desvio-padrão calculado entre as diferentes séries. Para Klesta \& Bartz (1996), os coeficientes de variação dentro da série (precisão) devem oscilar entre 5 e $10 \%$ para a etapa de dosagem. Segundo Raij et al. (2001), os coeficientes de variação entre as diferentes séries (reprodutibilidade) devem ser menores que $20 \%$. Esses valores foram adotados para interpretação dos resultados.

Para comparação dos métodos de dosagem, os tratamentos originaram-se do esquema fatorial $2 \times 36$, dispostos em blocos casualizados com quatro repetições. Os fatores corresponderam aos dois métodos de dosagem e aos 36 solos.

Foi aplicado também, para cada elemento, o procedimento estatístico proposto por Leite \& Oliveira (2002), que testa a identidade de dois métodos analíticos. Segundo os autores, os métodos são considerados estatisticamente iguais se, simultaneamente, após o ajuste da regressão linear, $\hat{\mathrm{Y}}=\beta_{0}+\beta_{1} \mathrm{X}+\varepsilon, \beta_{0}$ for igual a zero e $\beta_{1}$ for igual a 1 , estando o valor de $r$ próximo a 1 . Para testar as hipóteses $\mathrm{H}_{0}$ : $\beta^{\prime}=\left[\begin{array}{ll}0 & 1\end{array}\right], \mathrm{H}_{0}: \overline{\mathrm{e}}=0$ e avaliar o coeficiente de correlação linear $r$, o procedimento combina a estatística F, modificada por Graybill (1976), o teste do erro médio, que indica se as diferenças ocorreram ao acaso, e o coeficiente de correlação linear. Com base nessas informações, seguiu-se a regra decisória para testar a hipótese de identidade entre os dois métodos (Quadro 2).

\section{Quadro 2. Regra de decisão para comparação dos métodos ICP e EAA}

\begin{tabular}{|c|c|c|c|c|}
\hline Situação & $\mathrm{F}\left(\mathrm{H}_{0}\right)$ & $\mathbf{t}_{\overline{\mathrm{e}}}$ & $\mathbf{r}$ & Decisão \\
\hline 1 & $\mathrm{~ns}$ & $\mathrm{~ns}$ & $\mathrm{r} \geq(1-|\overline{\mathrm{e}}|)$ & $\mathrm{ICP}=\mathrm{EAA}$ \\
\hline 2 & $\mathrm{~ns}$ & $\mathrm{~ns}$ & $\mathrm{r} \leq(1-|\overline{\mathrm{e}}|)$ & $\mathrm{ICP} \neq \mathrm{EAA}$ \\
\hline 3 & $\mathrm{~ns}$ & * & $\mathrm{r} \geq(1-|\overline{\mathrm{e}}|)$ & $\mathrm{ICP} \neq \mathrm{EAA}$ \\
\hline 4 & $\mathrm{~ns}$ & * & $\mathrm{r} \leq(1-|\overline{\mathrm{e}}|)$ & $\mathrm{ICP} \neq \mathrm{EAA}$ \\
\hline 5 & * & $\mathrm{ns}$ & $\mathrm{r} \geq(1-|\overline{\mathrm{e}}|)$ & $\mathrm{ICP} \neq \mathrm{EAA}$ \\
\hline 6 & * & $\mathrm{ns}$ & $\mathrm{r} \leq(1-|\overline{\mathrm{e}}|)$ & $\mathrm{ICP} \neq \mathrm{EAA}$ \\
\hline 7 & * & * & $\mathrm{r} \geq(1-|\overline{\mathrm{e}}|)$ & $\mathrm{ICP} \neq \mathrm{EAA}$ \\
\hline 8 & * & * & $\mathrm{r} \leq(1-|\overline{\mathrm{e}}|)$ & $\mathrm{ICP} \neq \mathrm{EAA}$ \\
\hline
\end{tabular}

ns e * denotam, respectivamente, não-significativo e significativo a $5 \%$.

Fonte: Leite \& Oliveira (2002). 


\section{RESULTADOS E DISCUSSÃO}

\section{Definição dos comprimentos de onda do ICP}

Na definição dos comprimentos de onda do ICP, constatou-se que, para os elementos Fe e Mn, extraídos por Mehlich-1, Mehlich-3 e DTPA-TEA, os picos de emissão apresentaram-se bem definidos, indicando baixa interferência de outros elementos, em todos os comprimentos de onda selecionados previamente. Quando se trabalhou com os extratores Mehlich-1 e DTPA-TEA, os picos de emissão mais uniformes ocorreram em 259,929 nm para o Fe e em 259,372 nm para o Mn (Quadro 3). Quando se utilizou o extrator Mehlich-3, a curva de calibração não apresentou bom ajuste para estes dois comprimentos de onda, razão pela qual eles não foram selecionados. Para leitura do extrato de Mehlich-3, foram selecionados os comprimentos de onda de 234,349 nm para Fe e 260,568 nm para Mn (Quadro 3).

Para $\mathrm{Zn}$ e $\mathrm{Cu}$, em virtude das baixas leituras dos teores normalmente encontrados nos solos, as emissões apresentaram-se com muitas interferências, independentemente do extrator utilizado. A escolha do comprimento de onda, para esses dois elementos, foi priorizada pelo critério da máxima emissão ótica, sendo selecionados, para Mehlich-1, Mehlich-3 e DTPA-TEA, os comprimentos de onda de 213,857 e $324,752 \mathrm{~nm}$ para $\mathrm{Zn} \mathrm{e} \mathrm{Cu}$, respectivamente (Quadro 3).

A escolha de se trabalhar apenas no plano de visão axial deveu-se ao fato de isso agilizar as análises, pois, quando se trabalha nos dois planos de visão, as leituras deixam de ser simultâneas, aumentando o tempo, o consumo de gás e o custo da análise.

\section{Precisão e reprodutibilidade dos métodos de dosagem}

Com Mehlich-1 (Quadro 4), o ICP apresentou boa precisão e reprodutibilidade, com exceção da dosagem de $\mathrm{Zn}$ do solo $\mathrm{C}$, em que o CV foi maior que $10 \%$ na avaliação da precisão. O EAA foi preciso e reprodutível para $\mathrm{Fe}$ e Mn. Para $\mathrm{Zn}$ e $\mathrm{Cu}$, o EAA foi preciso e reprodutível apenas para o solo A. Em razão do menor limite de detecção, o ICP mostrou-se mais preciso e reprodutível que o EAA, principalmente nas dosagens de $\mathrm{Zn}$ e $\mathrm{Cu}$ em baixas concentrações.

Com Mehlich-3 (Quadro 5), tanto o ICP quanto o EAA apresentaram boa precisão e reprodutibilidade nas dosagens de Fe e Mn. Nas dosagens de Zn do solo $\mathrm{C}$ e de $\mathrm{Cu}$ do solo B, o ICP foi pouco preciso e pouco reprodutível, respectivamente. O EAA foi pouco preciso nas dosagens de $\mathrm{Zn}$ do solo $\mathrm{C}$ e de $\mathrm{Cu}$ dos três solos. Quanto à reprodutibilidade do EAA, ela foi baixa para Zn do solo C e para Cu dos solos B e C. Observouse, novamente, perda de precisão e reprodutibilidade mais acentuada no EAA, nos solos com teores mais baixos de $\mathrm{Zn}$ e $\mathrm{Cu}$.

Com DTPA-TEA (Quadro 6), os métodos de dosagem ICP e EAA apresentaram boa precisão e reprodutibilidade para os micronutrientes analisados. Observou-se exceção para o elemento Zn do solo C, em razão de a concentração ter sido de apenas $0,2 \mathrm{mg} \mathrm{dm}^{-3}$ do elemento.

\section{Comparação dos métodos de dosagem}

Para comparar os métodos de dosagem, foram consideradas 120 observações, das 144 possíveis (36 x 4), eliminando as leituras das amostras com teores extremamente altos. Com isso, objetivou-se verificar o comportamento dos métodos de dosagem para teores normalmente encontrados nos solos brasileiros. Dessa forma, aplicou-se o teste de identidade entre métodos analíticos (Leite \& Oliveira, 2002) e foram confeccionados gráficos da relação entre teores dosados pelo ICP e pelo EAA (Figura 1).

Na dosagem de Fe em Mehlich-1, o ICP, em relação ao EAA, apresentou erros sistemáticos $\left(B_{0} \neq 0\right)$, e as diferenças entre os métodos de dosagem não ocorreram ao acaso $\left(t_{\bar{\varepsilon}}>t_{\alpha}(n-1)\right)$. Em Mehlich-3, o ICP apresentou erros sistemáticos e baixa correlação (Figura 1). Em DTPA-TEA foram constatados erros proporcionais nas dosagens realizadas no ICP.

Para Zn, o ICP apresentou erros sistemáticos, independentemente do extrator utilizado. Houve

Quadro 3. Comprimentos de onda selecionados para leitura de Fe, Zn, Cu e Mn no plano axial do ICP, de acordo com o método de extração

\begin{tabular}{ccccc}
\hline Método & Fe & Zn & Cu & Mn \\
\hline & & & & \\
\cline { 2 - 4 } & 259,939 & 213,857 & 324,752 & 259,372 \\
Mehlich-1 & 234,349 & 213,857 & 324,752 & 260,568 \\
DTPA-TEA & 259,939 & 213,857 & 324,752 & 259,372 \\
\hline
\end{tabular}


Quadro 4. Média, precisão e reprodutibilidade dos métodos de dosagem dos nutrientes de três solos extraídos com Mehlich-1, em 10 séries analíticas, com três repetições por série

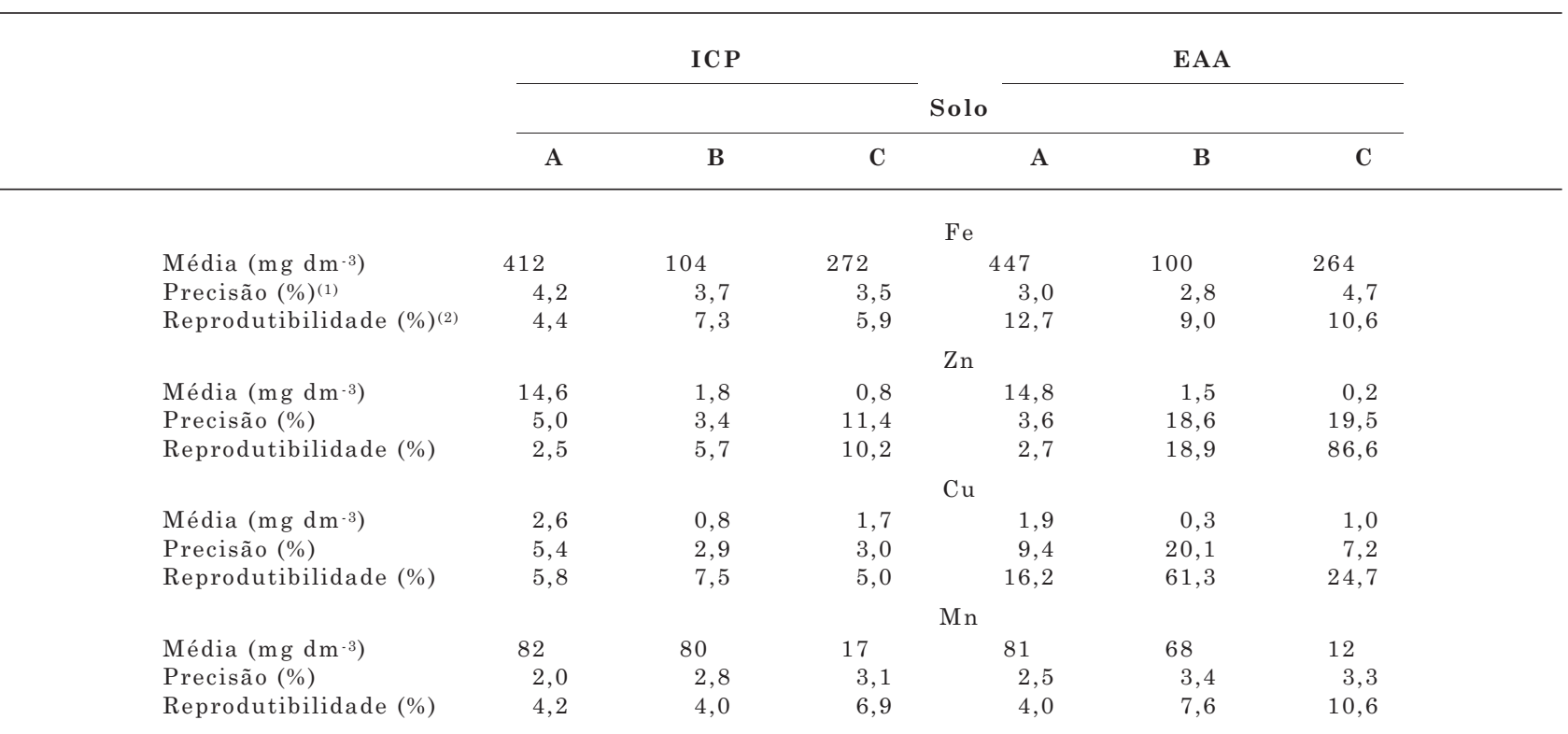

(1) Precisão: coeficiente de variação dos resultados dentro da mesma série analítica. ${ }^{\left({ }^{2}\right)}$ Reprodutibilidade: coeficiente de variação dos resultados entre as séries analíticas.

Quadro 5. Média, precisão e reprodutibilidade dos métodos de dosagem de Fe, Zn, Cu e Mn de três solos extraídos com Mehlich-3, em 10 séries analíticas, com três repetições por série

\begin{tabular}{ccccccc} 
& ICP & & \multicolumn{3}{c}{ EAA } \\
\cline { 6 - 7 } & & & Solo & & & \\
\hline A & B & C & A & B & C
\end{tabular}

\begin{tabular}{|c|c|c|c|c|c|c|}
\hline \multirow[b]{2}{*}{ Média $\left(\mathrm{mg} \mathrm{dm}^{-3}\right)$} & \multicolumn{6}{|c|}{$\mathrm{Fe}$} \\
\hline & 365 & 152 & 222 & 386 & 132 & 214 \\
\hline Precisão $(\%)^{(1)}$ & 2,9 & 2,3 & 2,4 & 3,5 & 2,3 & 2,4 \\
\hline \multirow[t]{2}{*}{ Reprodutibilidade $(\%)^{(2)}$} & 6,1 & 3,1 & 3,1 & 10,8 & 16,2 & 11,5 \\
\hline & \multicolumn{6}{|c|}{$\mathrm{Zn}$} \\
\hline Média $\left(\mathrm{mg} \mathrm{dm}^{-3}\right)$ & 9,0 & 1,2 & 0,7 & 9,2 & 0,8 & 0,04 \\
\hline Precisão (\%) & 3,9 & 4,7 & 15,8 & 2,2 & 12,5 & 81,7 \\
\hline \multirow[t]{2}{*}{ Reprodutibilidade (\%) } & 2,3 & 8,1 & 15,3 & 5,4 & 14,3 & 120,1 \\
\hline & \multicolumn{6}{|c|}{$\mathrm{Cu}$} \\
\hline Média $\left(\mathrm{mg} \mathrm{dm}^{-3}\right)$ & 0,8 & 0,4 & 0,5 & 1,3 & 0,3 & 0,5 \\
\hline Precisão (\%) & 4,0 & 5,4 & 4,5 & 14,4 & 28,1 & 12,9 \\
\hline \multirow[t]{2}{*}{ Reprodutibilidade (\%) } & 11,2 & 25,1 & 19,0 & 12,5 & 73,2 & 42,1 \\
\hline & \multicolumn{6}{|c|}{$\mathrm{Mn}$} \\
\hline Média $\left(\mathrm{mg} \mathrm{dm}^{-3}\right)$ & 49 & 36 & 7 & 48 & 35 & 6 \\
\hline Precisão (\%) & 3,2 & 4,0 & 3,7 & 2,4 & 3,0 & 5,2 \\
\hline Reprodutibilidade (\%) & 2,7 & 2,6 & 11,0 & 3,6 & 6,1 & 13,4 \\
\hline
\end{tabular}

(1) Precisão: coeficiente de variação dos resultados dentro da mesma série analítica. ${ }^{(2)}$ Reprodutibilidade: coeficiente de variação dos resultados entre as séries analíticas.

também diferenças tendenciosas nas dosagens de Zn em Mehlich-1 e em Mehlich-3 (Quadro 7).
Para Cu extraído com Mehlich-1 e com Mehlich-3, observaram-se erros sistemáticos, proporcionais 
Quadro 6. Média, precisão e reprodutibilidade dos métodos de dosagem dos nutrientes de três solos extraídos com DTPA-TEA, em 10 séries analíticas, com três repetições por série

\begin{tabular}{|c|c|c|c|c|c|c|}
\hline & \multicolumn{3}{|c|}{ ICP } & \multicolumn{3}{|c|}{ EAA } \\
\hline & A & B & $\mathrm{C}$ & A & B & $\mathrm{C}$ \\
\hline & \multicolumn{6}{|c|}{$\mathrm{Fe}$} \\
\hline Média $\left(\mathrm{mg} \mathrm{dm}^{-3}\right)$ & 115 & 57 & 89 & 117 & 56 & 88 \\
\hline Precisão $(\%)^{(1)}$ & 3,7 & 1,5 & 2,2 & 3,0 & 1,7 & 2,5 \\
\hline \multirow[t]{2}{*}{ Reprodutibilidade $(\%)^{(2)}$} & 10,1 & 4,7 & 5,0 & 12,7 & 9,9 & 13,2 \\
\hline & \multicolumn{6}{|c|}{$\mathrm{Zn}$} \\
\hline Média $\left(\mathrm{mg} \mathrm{dm}^{-3}\right)$ & 6,8 & 0,4 & 0,2 & 6,4 & 0,5 & 0,2 \\
\hline Precisão (\%) & 1,7 & 10,5 & 30,3 & 2,3 & 17,0 & 34,4 \\
\hline \multirow[t]{2}{*}{ Reprodutibilidade (\%) } & 4,3 & 13,3 & 39,0 & 3,3 & 14,6 & 61,0 \\
\hline & \multicolumn{6}{|c|}{$\mathrm{Cu}$} \\
\hline Média (mg dm-3) & 1,7 & 0,9 & 1,4 & 1,7 & 0,9 & 1,3 \\
\hline Precisão (\%) & 4,8 & 2,4 & 3,9 & 6,2 & 8,3 & 5,8 \\
\hline \multirow[t]{2}{*}{ Reprodutibilidade (\%) } & 7,0 & 7,5 & 4,8 & 8,3 & 10,8 & 10,1 \\
\hline & \multicolumn{6}{|c|}{$\mathrm{Mn}$} \\
\hline Média (mg dm³) & 13 & 17 & 3 & 13 & 17 & 3 \\
\hline Precisão (\%) & 4,7 & 1,4 & 2,2 & 4,4 & 1,5 & 3,0 \\
\hline Reprodutibilidade (\%) & 11,5 & 1,8 & 4,6 & 8,2 & 1,8 & 3,6 \\
\hline
\end{tabular}

(1) Precisão: coeficiente de variação dos resultados dentro da mesma série analítica. ${ }^{(2)}$ Reprodutibilidade: coeficiente de variação dos resultados entre as séries analíticas.
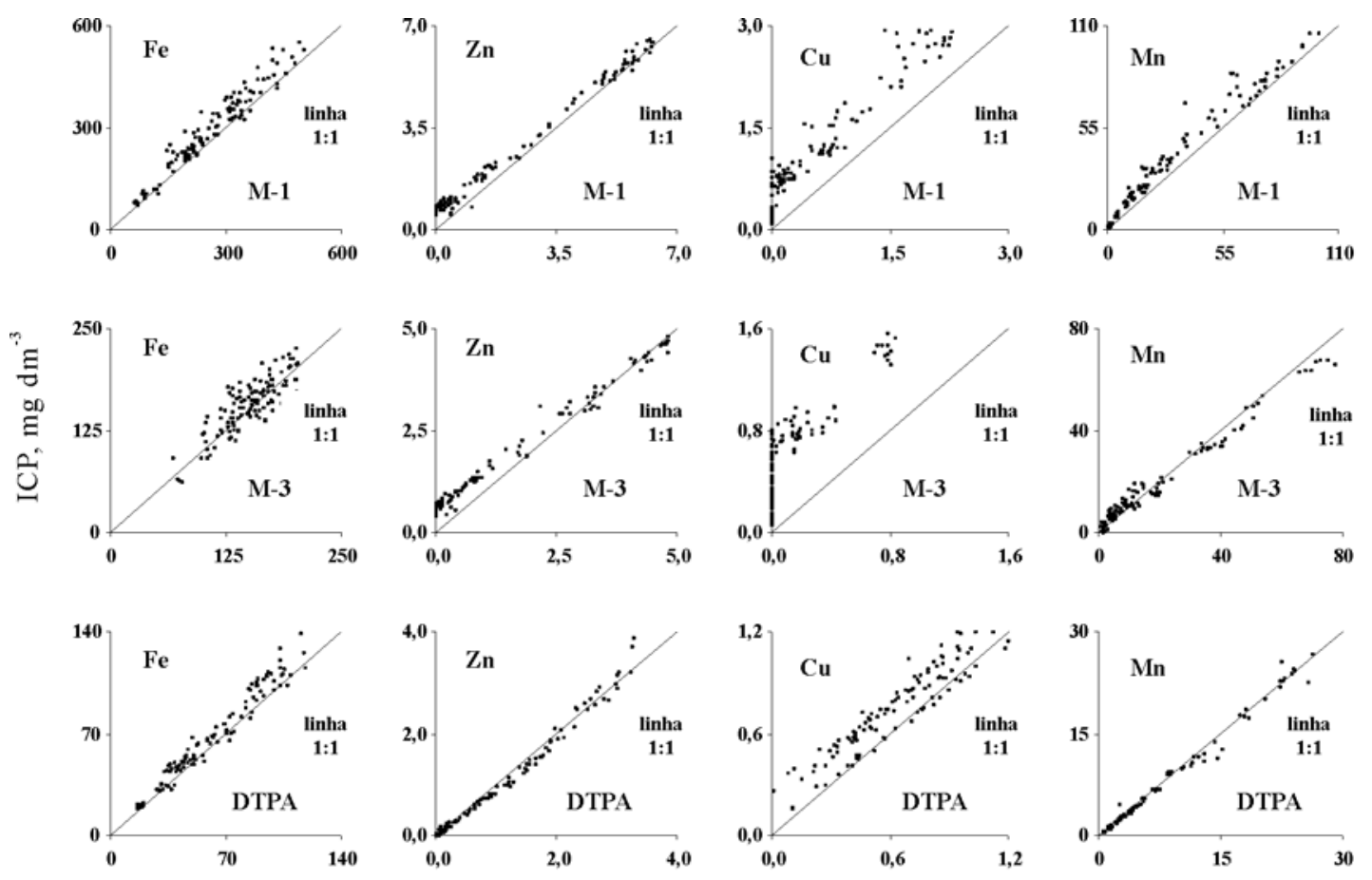

EAA, $\mathrm{mg} \mathrm{dm}^{-3}$

Figura 1. Relação entre os teores de Fe, Zn, Cu e Mn extraídos por Mehlich-1 (M-1), Mehlich-3 (M-3) e DTPA-TEA (DTPA) e dosados por espectrofotometria de emissão ótica em plasma induzido (ICP) e espectrofotometria de absorção atômica (EAA). 
Quadro 7. Resultados obtidos pela aplicação do teste de identidade entre métodos analíticos

Nutriente $\mathbf{n}^{(1)} \quad \mathbf{B}_{0}{ }^{(2)} \quad \mathbf{B}_{1}{ }^{(3)} \quad \mathbf{r}^{(4)} \quad \overline{\mathbf{e}}^{(5)} \quad \mathbf{F}\left(\mathrm{H}_{0}\right)^{(6)} \quad \mathbf{t}_{\bar{e}^{(7)}} \quad \mathbf{r} \geq(1-|\overline{\mathbf{e}}|) \quad$ Conclusão

\begin{tabular}{|c|c|c|c|c|c|c|c|c|c|}
\hline $\mathrm{Fe}$ & 120 & $17,4^{* *}$ & $1,0615^{\mathrm{ns}}$ & 0,97 & 0,1404 & $85,61 * *$ & $12,58^{* *}$ & Sim & $\operatorname{ICP}^{(8)} \neq \mathrm{EAA}^{(9)}$ \\
\hline $\mathrm{Zn}$ & 120 & $0,61^{* *}$ & $0,9218^{\mathrm{ns}}$ & 1,00 & $9.063,2563$ & $425,60 * *$ & $4,37 * *$ & Sim & $\mathrm{ICP} \neq \mathrm{EAA}$ \\
\hline $\mathrm{Cu}$ & 120 & $0,51^{* *}$ & $1,1220^{* *}$ & 0,96 & $11.327,0965$ & $371,24^{* *}$ & $5,39 * *$ & Sim & $\mathrm{ICP} \neq \mathrm{EAA}$ \\
\hline $\mathrm{Mn}$ & 120 & $5,6^{* *}$ & $1,0527 * *$ & 0,99 & $1.282,1533$ & $123,62 * *$ & $1,00^{\mathrm{ns}}$ & Sim & $\mathrm{ICP} \neq \mathrm{EAA}$ \\
\hline \multicolumn{10}{|c|}{ Mehlich-3 } \\
\hline $\mathrm{Fe}$ & 120 & $30,4^{* *}$ & $0,8285^{\mathrm{ns}}$ & 0,88 & 0,0310 & $10,48 * *$ & $2,59^{\mathrm{ns}}$ & Não & $\mathrm{ICP} \neq \mathrm{EAA}$ \\
\hline $\mathrm{Zn}$ & 120 & $0,58^{* *}$ & $0,8571^{\mathrm{ns}}$ & 0,99 & $14.031,7672$ & $491,65^{* *}$ & $6,31^{* *}$ & Sim & $\mathrm{ICP} \neq \mathrm{EAA}$ \\
\hline $\mathrm{Cu}$ & 120 & $0,42^{* *}$ & $1,4510^{* *}$ & 0,86 & $21.356,6882$ & 302,96 ** & $9,85^{* *}$ & Sim & $\mathrm{ICP} \neq \mathrm{EAA}$ \\
\hline $\mathrm{Mn}$ & 120 & $2,3^{* *}$ & $0,8820^{\mathrm{ns}}$ & 0,99 & 0,2551 & $58,99 * *$ & $4,23^{* *}$ & Sim & $\mathrm{ICP} \neq \mathrm{EAA}$ \\
\hline \multicolumn{10}{|c|}{ DTPA-TEA } \\
\hline $\mathrm{Fe}$ & 120 & $0,9^{\mathrm{ns}}$ & $1,0901^{* *}$ & 0,98 & 0,1129 & $67,62^{* *}$ & $11,65^{* *}$ & Sim & $\mathrm{ICP} \neq \mathrm{EAA}$ \\
\hline $\mathrm{Zn}$ & 120 & $-0,06 * *$ & $1,0172^{\mathrm{ns}}$ & 0,99 & 45,8610 & $8,15^{* *}$ & $1,38^{\mathrm{ns}}$ & $\mathrm{Sim}$ & $\mathrm{ICP} \neq \mathrm{EAA}$ \\
\hline $\mathrm{Cu}$ & 120 & $0,17 * *$ & $0,9328^{\mathrm{ns}}$ & 0,95 & 0,7037 & $115,57 * *$ & $1,68^{\mathrm{ns}}$ & Sim & $\mathrm{ICP} \neq \mathrm{EAA}$ \\
\hline $\mathrm{Mn}$ & 120 & $-0,1^{\mathrm{ns}}$ & $0,9954^{\mathrm{ns}}$ & 0,99 & $-0,0424$ & $4,13^{\mathrm{ns}}$ & $-3,67 * *$ & Sim & $\mathrm{ICP} \neq \mathrm{EAA}$ \\
\hline
\end{tabular}

(1) Número de observações. ${ }^{(2)}$ Intercepto da regressão. ${ }^{(3)}$ Coeficiente da regressão linear. ${ }^{(4)}$ Coeficiente de correlação linear simples. ${ }^{(5)}$ Erro médio. ${ }^{(6)}$ Teste F. ${ }^{(7)}$ Teste t para o erro médio. ${ }^{(8)}$ Espectrofotometria de emissão ótica em plasma induzido. ${ }^{(9)}$ Espectrofotometria de absorção atômica. ${ }^{\text {ns }} \mathrm{e}^{* *}$, não-significativo e significativo a $1 \%$, respectivamente.

$\left(\beta_{1} \neq 1\right)$ e diferenças tendenciosas do ICP em relação ao EAA (Quadro 7). Observou-se, também, que as dosagens deste elemento, principalmente em baixas concentrações, apresentaram-se bastante discrepantes, possivelmente em virtude de o ICP possuir um limite de detecção mais baixo que o do EAA (Figura 1). Em DTPA-TEA foram observados, apenas, erros sistemáticos, e as diferenças entre as dosagens ocorreram ao acaso.

Na dosagem do Mn extraído com Mehlich-1, embora as diferenças entre os métodos de dosagem tenham ocorrido ao acaso, foram constatados erros sistemáticos e proporcionais. Em Mehlich-3, a dosagem realizada no ICP apresentou erros sistemáticos, e as diferenças entre os métodos não ocorreram ao acaso. Na dosagem em DTPA-TEA, embora o ICP não tenha apresentado erros sistemáticos nem erros proporcionais, as diferenças entre os métodos não ocorreram ao acaso, razão pela qual se concluiu, pela regra de decisão (Quadro 2), que os métodos foram estatisticamente diferentes (Quadro 7).

Embora tenha havido alta correlação entre os valores obtidos no ICP e no EAA, exceto para Fe em Mehlich-3 ( $<<(1-|\overline{\mathrm{e}}|)$ (Quadro 7), os métodos de dosagem diferiram estatisticamente em todas as determinações.

\section{CONCLUSÕES}

1. Os comprimentos de onda que apresentaram as menores interferências no ICP foram: 259,939 nm para Fe em Mehlich-1 e DTPA-TEA e 234,349 nm em Mehlich-3; 213,857 nm para Zn e 324,752 nm para $\mathrm{Cu}$ nos três extratores; e 259,372 nm para Mn em Mehlich-1 e DTPA-TEA e 260,568 nm em Mehlich-3.

2. Nas dosagens de Fe e de Mn, os métodos ICP e EAA foram precisos e reprodutíveis para os três extratores utilizados. Nas dosagens de $\mathrm{Zn}$ e $\mathrm{Cu}$, o ICP mostrou ser mais preciso e reprodutível, principalmente quando os teores dos elementos apresentaram-se baixos. Esse fato pode ser explicado pelo menor limite de detecção do ICP em relação ao EAA.

3. O método ICP para a dosagem de $\mathrm{Fe}, \mathrm{Zn}, \mathrm{Cu}$ e Mn, extraídos por Mehlich-1, Mehlich-3 e DTPA-TEA, foi estatisticamente diferente do método EAA. Essa constatação compromete a interpretação dos resultados gerados pelo $\mathrm{ICP}$, com base nos níveis críticos gerados a partir do EAA.

\section{LITERATURA CITADA}

BOSS, C.B. \& FREDEEN, K.J. Concepts, instrumentation and techniques in inductively coupled plasma optical emission spectrometry. New York, Perkin Elmer, 1997.110p. 
COMISSÃO DE FERTILIDADE DO SOLO - CFSRS/SC Recomendações de adubação e de calagem para os Estados do Rio Grande do Sul e de Santa Catarina. 3.ed. Passo Fundo, SBCS/Núcleo Regional, 1994.

COMISSÃO DE FERTILIDADE DO SOLO DO ESTADO DE MINAS GERAIS - CFSEMG. Recomendação para o uso de corretivos e fertilizantes em Minas Gerais - $5^{\text {a }}$ Aproximação. Viçosa, MG, 1999. 359p.

DEFILIPO. B.V. \& RIBEIRO, A.C. Análise química do solo metodologia. 2.ed. Viçosa, MG, Universidade Federal de Viçosa, 1997. 26p.

GRAYBILL, F.A. Theory and application of the linear model. Belmont, Duxbury Press, 1976.

KLESTA, E.J. \& BARTZ, J.K. Quality assurance and control. In: BARTELS, J.M. \& BIGHAM, J.M., eds. Methods of soil analysis. Part 3. Chemical methods. Madison, Soil Science Society of America, 1996. p.9-48.

LEITE, H.G. \& OLIVEIRA, F.H.T. Statistical procedure to test identity between analytical methods. Comm. Soil Sci. Plant Anal., 37:1105-1118, 2002.

MEHLICH, A. Mehlich-3 soil test extractant: A modification of Mehlich-2 extractant. Comm. Soil Sci. Plant Anal., 15:1409-1416, 1984.
RAIJ, B. van; ANDRADE, J.C.; CANTARELLA, H. \& QUAGGIO, J.A., eds. Análise química para avaliação da fertilidade de solos tropicais. Campinas, Instituto Agronômico, 2001. 285p.

RAIJ, B. van; CANTARELLA, H.; QUAGGIO, J.A. \& FURLANI, A.M.C., eds. Recomendações de adubação e calagem para o Estado de São Paulo. 2.ed. Campinas, Instituto Agronômico \& Fundação, 1996. 285p. (Boletim Técnico, 100)

SOLTANPOUR, P.N.; WORKMAN, S.M. \& SCHWAB, A.P. Use of inductively-coupled plasma spectrometry for the simultaneous determination of macro and micronutrients in $\mathrm{NH}_{4} \mathrm{HCO}_{3}$-DTPA extracts of soils. Soil Sci. Soc. Am. J., 43:75-78, 1979 .

SOLTANPOUR, P.N.; JONES Jr, J.B. \& WORKMAN, S.M. Optical emission spectrometry. In: PAGE, A.L. Methods of soil analysis. Part 2. Chemical and microbiological properties. 2.ed. Madison, American Society of Agronomy, 1982. p.29-63.

VOCASEK, F.F. \& FRIEDERICKS, J.B. Soil micronutrient extraction by Mehlich-3 compared to $\mathrm{CaCl}_{2}$-DTPA. Comm. Soil Sci. Plant Anal., 25:1583-1593, 1994.

WILLETT, I.R. \& ZARCINAS, B.A. Nitric acid dissolution and multi-element analysis of soil and sediments by inductively coupled plasma spectrometry. Comm. Soil Sci. Plant Anal., 17:183-193, 1986. 